\title{
Novel Workflow for Improved Throughput, Turnaround Time, and Cross Section Preparation of Microelectronic Devices
}

William Podrazky ${ }^{1}$, Jamil Clarke ${ }^{1}$, Takeshi Sunaoshi ${ }^{1}$, Akinari Morikawa ${ }^{2}$, Chihiro Nomaguchi ${ }^{2}$ and Atsushi Muto ${ }^{1}$

${ }^{1}$ Hitachi High Technologies America, Inc, Clarksburg, Maryland, United States, ${ }^{2}$ Hitachi High Tech Corporation, Hitachinaka, Ibaraki, Japan

In the pursuit of high-quality scanning electron micrographs, proper sample preparation plays a key role. Traditional sample preparation techniques such as metallographic grinding and polishing are well developed; however these manual approaches can present challenges when the true microstructure and/or microchemistry of the sample is obscured or altered due to the use of inappropriate technique [1].

Additional, more automated, techniques are available to prepare samples with a high degree of quality and mitigate preparation-induced artifacts. One such approach is the utilization of a focused ion beam (FIB) instrument to produce cross sections of a sample region measuring up to $200 \mu \mathrm{m}$ in length and $20 \mu \mathrm{m}$ in depth [1]. Due to region of interest (ROI) size being limited, this method is best suited for situations where specific target areas can be easily defined [1]. An advantage of FIB cross section preparation is the ability to reveal very small voids or other fragile features without the potential damage or artifact induction involved in conventional sectioning techniques using mechanical means [1]. A related but differentiated class of ion-based approaches is to utilize broad ion beam (BIB) milling whereby ions, similar to FIBs, are used to prepare cross sections free from the damage experienced when using conventional mechanical techniques, however, this technique allows for addressing a much larger area (up to millimeters in length and depth) with faster material removal rates.

In this work, a novel approach to increase the speed, quality and site-specificity of preparing microelectronic device samples for analysis will be presented. This multi-faceted method combines ionbased techniques to more effectively address semiconductor components and microelectronic devises by utilizing BIB milling for site-specific large area bulk material removal and FIB for final polishing. Thus, improving the throughput and turnaround time (TAT) of samples prepared for scanning electron microscope (SEM) evaluation. This is crucial for process monitoring of common microelectronic components, such as ceramic capacitors, as presented here. The application of this workflow to address high aspect ratio semiconductor structures, such as through silicon vias (TSVs), will also be demonstrated.

The ceramic capacitor on a printed circuit board was attached to the ion milling stub using conductive tape and cross sectioned using a Hitachi ArBlade 5000 BIB milling system. Images of the target were acquired using the Hitachi SU5000 Field Emission Variable Pressure Scanning Electron Microscope (FEVP-SEM) before and after final milling as shown in Figure 1 and Figure 2. Final milling was performed using the Hitachi NX5000 FIB.

Figure 1 demonstrated SEM images of a cross section created after 60min of BIB milling. In Figure 1b, an area of $850 \mu \mathrm{m}$ wide by $650 \mu \mathrm{m}$ deep is shown to be sufficient for cross section visualization of the entire ceramic capacitor without any physical distortion (as confirmed by Figure 1c). Figure 2 shows SEM images of the cross section shown in Figure 1 following 10min of FIB polishing and demonstrates fine internal features, such as voids and intermetallic diffusion, with no alterations to the crystallographic microstructure. 

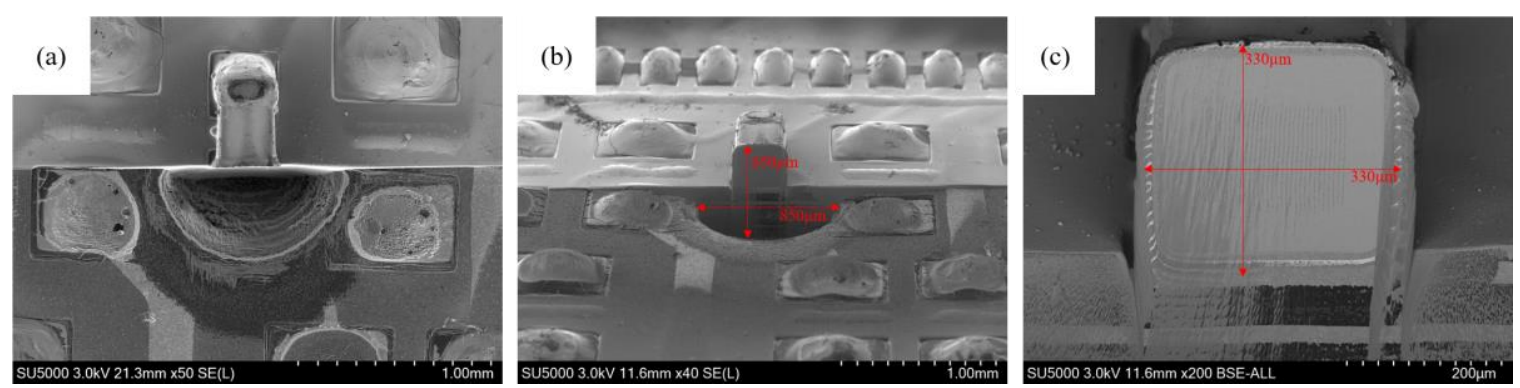

Figure 1. Results after BIB milling; (a) Top-down SEM image of a resistor cross section collected at $3 \mathrm{kV}$ accelerating voltage and 50X magnification. (b) Annotated cross section view of a resistor collected at $3 \mathrm{kV}$ accelerating voltage and $40 \mathrm{X}$ magnification. (c) Annotated cross section view of a resistor collected at $3 \mathrm{kV}$ accelerating voltage and $200 \mathrm{X}$ magnification.
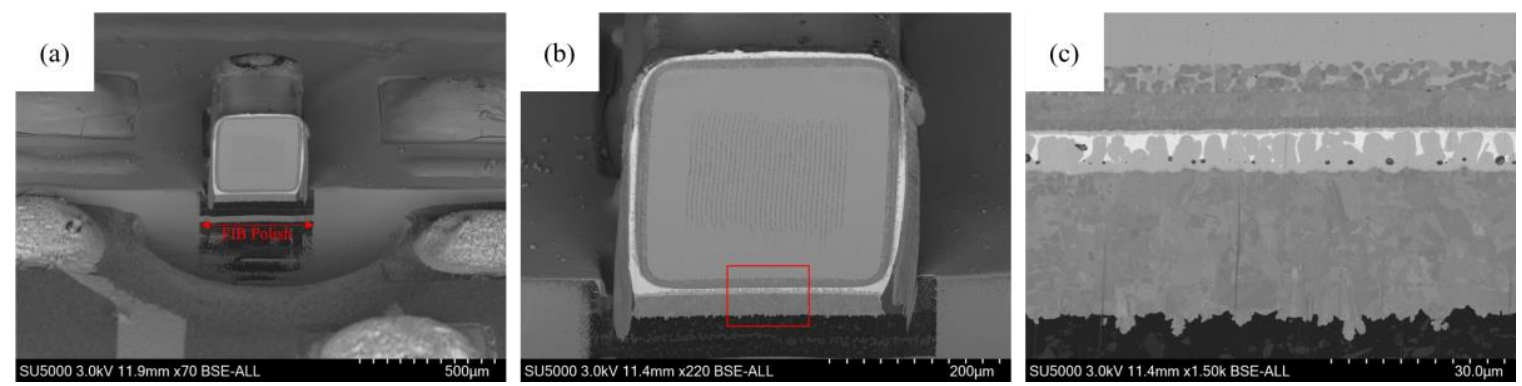

Figure 2. Results after FIB polishing; (a) Annotated cross section view a resistor collected at $3 \mathrm{kV}$ accelerating voltage and $70 \mathrm{X}$ magnification. (b) Annotated cross section view a resistor collected at $3 \mathrm{kV}$ accelerating voltage and 220X magnification. (c) Higher magnification investigation of the indicated portion of a resistor shown in (b) collected at $3 \mathrm{kV}$ accelerating voltage and 1,500X magnification.

References

[1] J. Goldstein et al., "Scanning Electron Microscopy and X-ray Microanalysis", (2003) p.p. 537-555 